057

\section{MANAGEMENT AND OUTCOME OF OUT-OF-HOSPITAL CARDIAC ARREST AT A MAJOR UK TERTIARY CENTRE}

F Jouhra, J Sapontis, S Leonard, N Melikian, J Byrne, R Dworakowski, A Shah, J Hill, M Whitbread, P MacCarthy King's College Hospital

doi:10.1136/heartjnl-2013-304019.57

Background Out of hospital cardiac arrest (OOHCA) is an increasingly common clinical conundrum for the interventional cardiologist, largely because paramedic resuscitation is becoming more effective. The 2011-2012 audit of the London Ambulance Service showed an increase in survival to hospital in patients with return of spontaneous circulation (ROSC) from $14 \%$ in $2007 / 2008$ to $30 \%$ in $2011 / 2012$. We analysed the survival of our cardiac arrest survivors and how eventual diagnosis impacted on outcome.

Methods A retrospective analysis of intensive therapy unit (ITU) data over a 20 month period was performed. Patients were either admitted through the Emergency Department or taken directly to the catheterisation laboratory depending on the presence of ST elevation on the presenting ECG. Those attending the catheter laboratory received primary revascularisation (PR) if appropriate. From a variety of sources we obtained survival rates in ITU, hospital and at 30 day post-discharge.

Results 108 patients with OOHCA were admitted to our institution during the study period. Of these $55(51 \%)$ patients were admitted via the A\&E and 53 (49\%) were taken straight to the cath lab. Of the OOHCA taken to the cath lab all patients underwent coronary angiography. PR was performed only on OOHCA patients with conclusive evidence of an occluded vessel 44/108 (41\%). The total number of OOHCA that left ITU alive irrespective of cause was 55/108 (51\%). The number of OOHCA

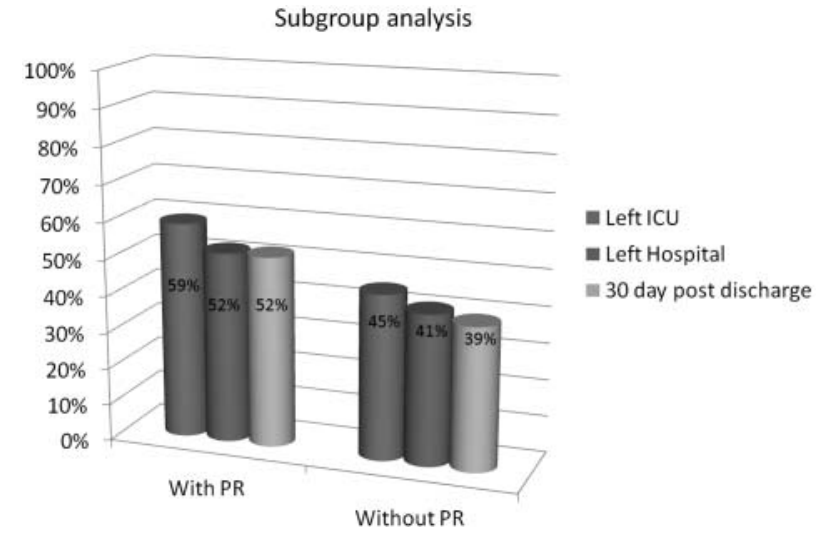

Figure 2

patients who left ITU after PR was 26/44 (59\%) compared with those that did not receive PR 29/64 (45\%). The total number of OOHCA patients who left hospital was $49 / 108$ (45\%). The total number of patients with PR who left hospital was 23/44 (52\%) and those who received no PR was 26/64 (41\%). The overall survival at 30 days for OOHCA at $\mathrm{KCH}$ was 48/108 (44\%). The 30 day survival for those patients receiving PR was 23/44 (52\%) and those not receiving PR was 25/64 (39\%). The eventual diagnosis in the no ST elevation group was extremely varied and included cardiac and non-cardiac aetiologies.

Discussion Improved effectiveness of paramedic resuscitation is increasing the number of OOHCA survivors with ROSC delivered to all major cardiac centres. This is a growing cohort of patients and consensus about optimal care pathways has yet to be reached in the UK. Overall survival in this cohort was higher than expected. It seems from these data that directing patients according to the presence of ST elevation in the ECG is reasonable. Those patients with ST elevation undergoing PR have the best prognosis.

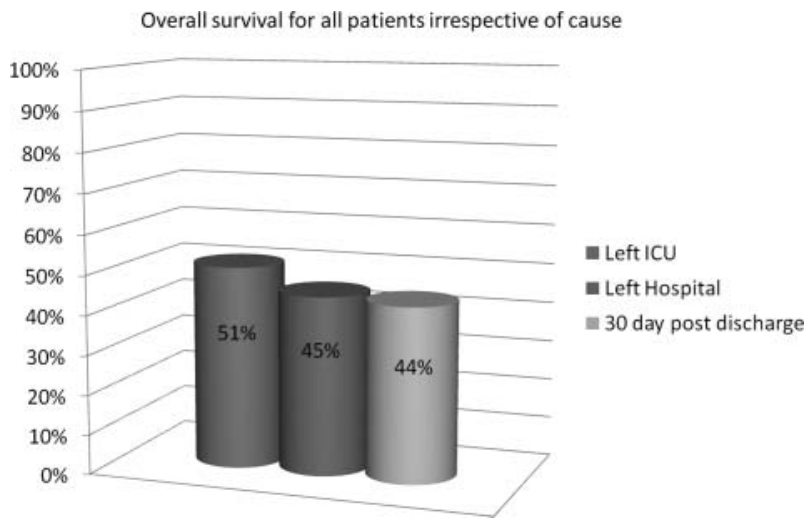

Figure 1 